Service social

\title{
Par-delà nos frontières. Le développement d'alliances par le travail de groupe
}

Jocelyn Lindsay et Daniel Turcotte

Volume 46, numéro 2-3, 1997

Groupes - Symposium 1997

URI : https://id.erudit.org/iderudit/706755ar

DOI : https://doi.org/10.7202/706755ar

Aller au sommaire du numéro

Éditeur(s)

École de service social de l'Université Laval

ISSN

1708-1734 (numérique)

Découvrir la revue

Citer ce document

Lindsay, J. \& Turcotte, D. (1997). Par-delà nos frontières. Le développement

d'alliances par le travail de groupe. Service social, 46(2-3), 5-9.

https://doi.org/10.7202/706755ar d'utilisation que vous pouvez consulter en ligne.

https://apropos.erudit.org/fr/usagers/politique-dutilisation/ 


\section{AVANT.PROPOS}

\section{Par-delà nos frontières Le développement d'alliances par le travail de groupe}

Ce titre reprend le thème du XIXe Symposium annuel de l'Association pour le développement du service social des groupes, qui s'est tenu à Québec du 23 au 26 octobre 1997. À cette occasion, plus de 400 personnes, provenant de treize pays, ont « franchi leurs frontières " et se sont réunies pendant quatre jours pour discuter de service social des groupes dans des séances plénières, des ateliers de formation, des présentations spéciales, des classes des maîtres, ainsi que dans des communications et des ateliers de pratique. Nous allons ici développer la raison d'être de ce thème (choisi par concours lors d'une précédente journée Simone-Paré) et présenter le contenu des Actes.

Franchir des frontières et établir des alliances font partie du quotidien des travailleurs sociaux de groupe : développer l'entraide entre les personnes est le fondement même du travail de groupe; mais aussi n'arrive-t-il pas qu'il faille franchir des frontières, et parfois des barrières, quand arrive le temps de faire accepter un projet de groupe comme un outil pertinent pour la pratique sociale?

Si l'on se place à un autre niveau, on constate que les conditions présentes sur les plans technologique, économique et social créent de nouveaux problèmes à résoudre et aussi des possibilités de redéfinir notre champ professionnel. L'établissement d'alliances est un moyen important pour réduire les limites auxquelles nous sommes confrontés et pour bâtir un avenir plus prometteur.

Mais comment concrétiser ce développement d'alliances par-delà le travail de groupe dont il est une caractéristique essentielle? Sans 
prétendre décrire de façon exhaustive les différentes facettes de cette question, nous pouvons au moins mentionner certaines stratégies par lesquelles les travailleurs sociaux concrétisent régulièrement le développement d'alliances, et cela a fait l'objet de plusieurs présentations.

Un premier aspect est celui de la dimension multiculturelle du travail, afin de dépasser l'isolement et la confrontation avec les populations qu'on dessert. Un second aspect est le suivant : comment le service social des groupes peut-il s'allier à d'autres formes de connaissance, par exemple les arts comme la musique ou la peinture, pour contribuer à une intervention plus efficace? Également, la jonction des processus de recherche et d'intervention peut apporter des dividendes. À un niveau organisationnel, le partenariat entre les organismes de pratique et les équipes de recherche peut aussi profiter aux uns et aux autres. Sur le plan de la formation, les stages constituent un élément majeur de la formation en service social des groupes et ils nécessitent une collaboration entre les écoles et les organismes de pratique. Également, dans l'état actuel de développement du service social des groupes, les écoles ont avantage à collaborer pour assurer une plus grande qualité de la formation. L'avènement de nouvelles technologies rend aussi possibles, par des moyens comme l'informatique ou les téléconférences, des discussions et des mises en commun qui auraient été impensables il y a une ou deux décennies. Enfin, une dernière forme d'alliance, et qui se vit au quotidien pour les intervenants engagés dans des interventions de groupe, a trait à la complémentarité entre les méthodes d'intervention en service social. Sans qu'elles aient été ici développées, voilà autant de possibilités ou de lieux dans lesquels on peut dépasser les frontières et établir des alliances.

Les premiers textes de ce numéro regroupent des présentations qui ont été faites dans les séances plénières. Au départ, Paul Taylor pose la question suivante : comment créer une praxis éducative du travail social de groupe, à un niveau international, engagée dans la lutte humaine et solidaire pour une société radicalement plus juste et qui, en même temps, respecte la différence et l'identité culturelle d'un groupe donné? Explorant la complexité de cette question, il identifie quelques-uns des échanges frontaliers constructifs entre l'international et l'inter-culturel. En réaction à cette communication, Bernadette Ngo Nkouth souligne deux préoccupations issues de la 
présentation précédente, soit la différence dans la définition des concepts et la faisabilité du principe de la réciprocité dans le modèle proposé.

Prenant comme exemple une intervention de groupe qu'il coanime auprès de personnes atteintes du sida en phase préliminaire de désintoxication, Lawrence Shulman développe l'idée de franchir nos frontières de différentes façons : partenariat entre les milieux de formation et de pratique, jonction des processus de recherche et d'intervention, collaboration entre le service social et le counseling, combinaison de l'aide mutuelle et de l'approche éducative.

Dans le cadre de la plénière de clôture sous le thème " Comment faciliter la réussite de nos interventions malgré un contexte difficile? ", David Ward émet l'opinion qu'on a actuellement tendance à réduire le travail de groupe à des programmes globaux qui nient l'individualité des personnes, alors qu'il faut un modèle de pratique en mesure de contrer l'exclusion sociale par l'égalité et la démocratie. À partir du programme d'action communautaire pour les enfants (PACE), André Beaudoin décrit comment l'intervention de groupe représente une voie d'action particulièrement pertinente pour venir en aide aux jeunes enfants et à la famille, dans le contexte de transformation qui marque cette fin de siècle.

Les deux textes qui suivent mettent l'accent sur les développements théoriques en intervention de groupe. Soutenant que l'ampleur des problèmes sociaux requiert une plus grande polyvalence de la part des intervenants, Jean-Pierre Deslauriers et Monique Bourget traitent des liens qui se sont tissés entre le travail de groupe et l'organisation communautaire, en prenant comme exemple l'implantation des cuisines collectives. Comme une proportion grandissante des interventions vise les populations qui sont généralement perçues comme réfractaires aux services sociaux, Daniel Turcotte précise en quoi l'approche systémique offre des repères utiles pour le recrutement des membres au niveau de l'intervention auprès des populations défavorisées et il présente des stratégies de recrutement utilisées dans les milieux de pratique.

Trois textes illustrent la contribution des collègues européens à ce symposium. En se situant dans le contexte de la Suisse, pays carrefour de trois cultures et de quatre langues nationales, Christiane Besson montre comment le travail social de groupe et l'intervention 
de réseaux révèlent des similitudes et des différences, ouvrant ainsi la voie à des stratégies combinées et originales. Assistante sociale et enseignante en France, Catherine Manson traduit bien, à travers plusieurs exemples de pratique, la place que peut occuper le travail social avec les groupes dans le développement d'alliances entre les usagers, les professionnels et les élus dans la construction d'une politique sociale locale. Également de France, Christophe Dalibert décrit comment, dans un contexte de supervision, des professionnelles en difficulté dans leur travail et non initiées au travail de groupe ont bénéficié de cette méthode pour la rédaction de projets de groupe à l'intention d'usagers avec lesquels elles travaillent.

Sur le plan du processus de formation, Ginette Berteau et Danielle Grégoire traitent de la relation superviseur-stagiaire dans une perspective d'empowerment et en montrent la complexité dans les interventions où le superviseur est l'instigateur du projet et où une ancienne membre du groupe prend la relève au niveau de l'intervention. Lyse Montminy aborde l'utilisation du travail de groupe comme moyen efficace pour relever le défi de l'adaptation des étudiants au milieu et à la culture universitaires.

Les trois textes qui suivent ont en commun l'utilisation du travail de groupe avec des types particuliers de clientèle. Pierre Turcotte présente sa recherche dans laquelle il tente de clarifier la place qu'occupe le contrôle social dans les thérapies de groupe pour conjoints agresseurs. Claudine Parent et Madeleine Beaudry décrivent les fondements théoriques et les principales composantes d'un programme d'intervention préventive conçu pour les couples qui vivent la recomposition de leur famille. En s'appuyant sur sa pratique, Natalie Bibeau montre qu'il est possible d'adapter l'intervention de groupe aux besoins des hommes et des femmes présentant des problèmes d'alcoolisme ou de toxicomanie.

Quatre courts textes ont enfin été colligés comme autant d'illustrations de pratique s'inscrivant bien dans le cadre du thème du Symposium. Marie Berlinguet et Nicole Gagné décrivent une expérience de groupe interdisciplinaire auprès de familles d'accueil pour adolescents en prévention des MTS-VIH/SIDA au Centre jeunesse de Québec; ce programme a su intégrer les connaissances et les expertises de plusieurs disciplines. Dans une expérience d'intervention auprès de personnes sans domicile fixe ou stable, Pascal 
Dabouis montre, à partir d'outils d'évaluation, l'efficacité du travail de groupe sur les parcours de ces personnes et sur la vie interne et externe d'un centre d'hébergement. Le texte de Jacques Beaulieu et de Bernard Levesque, qui œuvrent dans le secteur du maintien à domicile, porte sur les mécanismes de concertation entre partenaires ainsi que sur les résultats d'une démarche de transformation d'un groupe de soutien aux aidants naturels en un groupe d'entraide. S'inspirant de deux expériences, l'une québécoise et l'autre belge, Lorraine Filion montre comment une intervention de groupe auprès d'enfants vivant des transitions familiales peut normaliser le vécu de ces derniers, soulager leur détresse et favoriser l'émergence de solutions collectives à leurs problèmes.

Ces Actes n'incluent évidemment qu'une petite partie des 200 ateliers, présentations et communications qui se sont succédé dans le cadre du Symposium. Comme présidents du comité de coordination du Symposium et du comité de programme, nous avons été heureux qu'un tel événement, si riche de possibilités d'apprentissage et d'échanges interculturels et sociaux, ait lieu dans le charme historique de la ville de Québec. Merci aux organismes et aux nombreuses personnes qui, souvent dans l'ombre, ont rendu cette expérience possible. Nous espérons que ces Actes contribueront non seulement à enrichir les connaissances sur le service social des groupes, mais aussi à maintenir bien vivant le souvenir de fructueux échanges et de merveilleuses rencontres.

Jocelyn LINDSAY Daniel TURCOTTE 